\title{
The Main Molecules Involved in Human Mesenchymal Stem Cells Immunomodulation
}

\author{
Felipe de Lara Janz ${ }^{1, *}$, Helen Dutra Leite ${ }^{2}$, Sergio Paulo Bydlowski ${ }^{1}$ \\ ${ }^{1}$ Laboratory of Genetics and Molecular Hematology, University of Sao Paulo Medical School, Sao Paulo, SP, Brazil \\ ${ }^{2}$ Laboratory of Pharmaceutical Science, University of Sao Paulo, Sao Paulo, SP, Brazil \\ *Corresponding author: fljanz@usp.br
}

Received May 07, 2015; Revised May 28, 2015; Accepted June 05, 2015

\begin{abstract}
Mesenchymal stem cells (MSCs) are described as undifferentiated cells with high capacity for selfrenewal and differentiation ability in different tissues. MSCs are found in various locations in the adult organism as bone marrow, adipose tissue; and in fetal tissues as umbilical cord, placenta and amniotic fluid. They are able to produce and secrete a number of bioactive molecules with different effects: anti-fibrotic, angiogenic and mitogen. These cells also present a great immunomodulatory and anti-inflammatory potential described in experimental and human models. Several studies have demonstrated the ability of MSCs to suppress the proliferation and activation of $\mathrm{T}$, B and NK cells in vitro and in vivo. Its low immunogenic action causes are not recognized by HLA mismatched receptor complex because they express low levels of MHC-I do not express MHC-II and costimulatory molecules CD40, CD80 and CD86. Because of these unique characteristics, MSCs arouse great interest in possible clinical applications in the therapy of diseases that affect the immune system. Although depending on the tissue microenvironment, the MSCs can also trigger inflammatory events. In this work, we described the main factors and another structures involved in MSCs immunoregulation.
\end{abstract}

Keywords: mesenchymal stem cells, immunomodulation, soluble molecules

Cite This Article: Felipe de Lara Janz, Helen Dutra Leite, and Sergio Paulo Bydlowski, "The Main Molecules Involved in Human Mesenchymal Stem Cells Immunomodulation.” Biomedical Science and Engineering, vol. 3, no. 1 (2015): 4-8. doi: 10.12691/bse-3-1-2.

\section{Introduction}

Mesenchymal stem cells (MSCs) are considered adult multipotent cells with self-renewal and differentiation into mesenchymal tissues properties [1]. Therefore, MSC are an attractive cell-based therapy tool for developmental defects, degenerating diseases and bone, cartilage, muscle and other mesodermal tissues injuries. MSCs were initially isolated from bone marrow but can be found in almost every type of connective tissue. According to the International Society for Cellular Therapy a cell population is classified as mesenchymal stem cell when present three key features:

1) Must be plastic-adherent when cultured in standard protocols.

2) Phenotypically, ex vivo expanded MSCs, express a number of nonspecific markers, including CD105 (SH2 or endoglin), CD73 (SH3 or SH4), CD90, CD166, CD44, and CD29. MSC do not exhibit hematopoietic and endothelial markers, such as CD11b, CD14, CD31 and CD45.

3) Can be differentiated into bone, fat and cartilage tissue after appropriate stimulation [2].

MSCs have immunomodulatory properties and play important roles in modulating tolerance to transplants, autoimmune syndromes, tumor infiltration and maternal- fetal tolerance. However, the mechanisms involved in these actions are not fully understood [3]. Homing ability is another important characteristic of MSCs because it allows these cells migrate in response to biochemical signals. MSCs express several receptors and cell adhesion molecules that aid in migration and homing to target tissues (e.g. inflammation and injuries sites). Immunomodulatory effects and homing potential of MSCs may be used to treat autoimmune diseases and prevent rejection of organ transplants [4].

Human MSCs express low levels of major histocompatibility complex (MHC) class I antigens (A, B, C, E, F and G) and not present MHC class II (DP, DQ and DR types) and co-stimulatory molecules CD40, CD80, and CD86 [5]. Studies have shown the inhibitory effect of MSC on human lymphocytes in vitro and in vivo [6]. Early studies reported that MSC were able to suppress the activation and proliferation of $\mathrm{T}$ lymphocytes induced by mitogens and alloantigen as well as CD3 and CD8 antibodies [7]. MSCs also decrease differentiation, maturation and function of dendritic cells (DCs). DCs perform antigen presentation and have a key role in immunity and tolerance induction [8]. After co-cultive with MSCs, DCs did not express costimulatory molecules and decreased their ability to activate T lymphocytes. DCs cytokines secretion was also changed: TNF-alpha and interferon gamma production decreased and antiinflammatory, IL-10, was increased [9]. These immune 
properties explain the fact that MSCs do not have immunological restriction; so, demonstrate the same effects in cells of autologous and allogeneic origin [5]. There are a large number of studies demonstrating xenogenic transplantation of human MSC without athymic animal models use and/or immunosuppressive therapy $[10,11]$.

MSCs immunomodulatory potential can range depending on the source from which it was obtained. Roelen, 2009 [12] showed that MSCs obtained from fetal tissues, such as umbilical cord and amnion, showed a higher immunomodulatory effect than the adult stem cells from bone marrow and adipose tissue. Another factor that can influence in MSCs immunologic properties is the differentiation/maturation process. Yuan et al. [13] demonstrated that MSCs derived from human bone marrow enhanced their immunogenicity after chondrogenic tissue differentiation in vitro.

Many studies report an effector role of soluble substances (paracrine factors) in MSCs immunoregulation, since the separation of these cells and mononuclear blood cells by a semipermeable membrane does not affect the immunomodulatory activity [14]. These factors are released into exosomes (Figure 1), which are small membranous vesicles secreted by most cell types that participate in cell-cell communication [15]. There are no differences in terms of morphological features, isolation, and storage conditions between exosomes derived from MSCs and other sources [16]. In according to this idea, previous studies have demonstrated that the immunomodulatory capacity of MSCs against NK cells, cytotoxic $\mathrm{T}$ lymphocytes, gamma delta $\mathrm{T}$ cells and dendritic cells is mediated by a paracrine mechanism.

Once released into the extracellular environment, exosomes may interact with recipient cells via adhesion to the cell membrane mediated by lipid-ligand receptor interactions, internalization via endocytic uptake, or by direct fusion of the vesicles and cell membrane, which leads to the release of exosome content into the target cells.

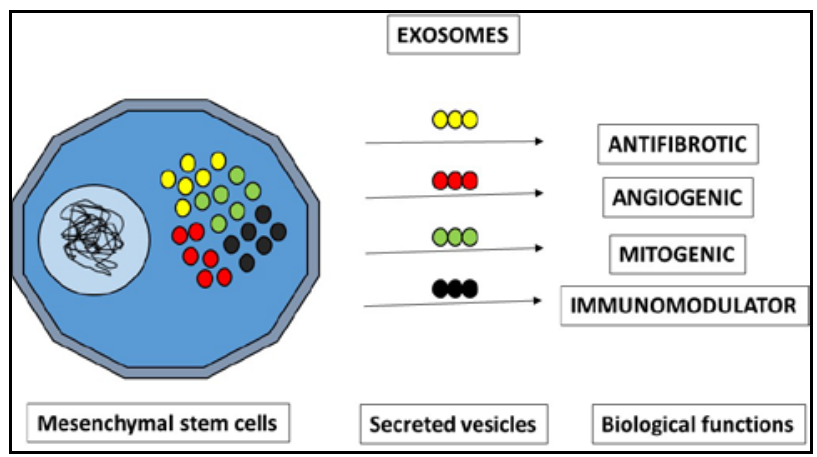

Figure 1. exosomes secreted by MSCs can promote several biological functions: antifibrotic, mitogenic, angiogenic and immunomodulatory effects

This paper aims to describe the main soluble substances secreted by human MSCs in the form of exosomes as well as showing another structures (receptors) involved in immunoregulation process.

\section{Prostaglandin E2 (PGE2)}

Prostaglandins are small-molecule derivatives of arachidonic acid, produced by cyclooxygenases (COX) and PG synthases, with a relatively contribution of the isoprostane pathway $[18,19]$. COX1 is constitutively expressed from virtually all tissues, while COX2 is induced under inflammatory conditions [20]. Several studies suggest that PGE2 subtype is responsible for immunosuppressive effects of MSCs.

PGE2 is constitutively secreted by MSCs and this production is increased when cocultived with $T$ lymphocytes [21]. LPS as well as cytokines like IFN- $\gamma$, TNF $\alpha$, IL-1 $\beta$ are mediators directly regulating PGE2 production from MSCs [22]. PGE2 selectively suppresses effector functions of macrophages and neutrophils and Th1-, CTL-, and NK cell-mediated type 1 immunity, but it promotes Th2, Th17, and regulatory $\mathrm{T}$ cell responses. PGE2 modulates chemokine production, inhibiting the attraction of proinflammatory cells while enhancing local accumulation of regulatory T cells [23]. Particularly, the cellular targets of PGE2 are PBMCs, NK cells, monocytes, macrophages and the transitional processes of monocytes into immature DCs differentiation [24].

\section{Indoleamine-2, 3-dioxygenase (IDO)}

The enzyme IDO that catabolizes tryptophan into Nformyl-kynurenine via cleavage of tryptophan's pyrrole ring and addition of an oxygen molecule is also described as suppressive molecule on T-cell proliferation by the MSCs. IDO is present in various tissues and its expression could be induced by certain infectious agents, lipopolysaccharides, and cytokines such as interferon- $\gamma$. In its normal physiologic action, IDO is important in modulating immune activation to antigenic challenges at mucosal surfaces in the digestive tract and lungs [25].

Not activated MSCs express low levels of IDO, but on stimulation with inflammatory cytokines, mainly interferon- $\gamma$, the IDO mRNA levels are found to be elevated [26]. The metabolites produced by IDO action are responsible for blocking $\mathrm{T}$ cells cycle [21]. This enzyme is also involved in the maintenance of maternal-fetal tolerance as a potent regulator of cell function in the immune system.

Induction of regulatory $\mathrm{T}$ cells is another indirect mechanism for immunoregulation explored by MSCs. IDO expression is responsible for induction of IL$10+/$ IFN $\gamma+/$ CD4+ regulatory T type 1 cells by MSC [27]. Evidence suggests that dendritic cells expressing IDO interact with regulatory $\mathrm{T}$ cells creating a microenvironment that favors tolerance induction [28].

\section{Human Leukocyte Antigen-G5 (HLA-G5)}

HLA-G molecules are classified as non-classical HLA-I proteins due to the following characteristics: limited allelic polymorphism; peculiar expression pattern characterized by the generation of seven protein isoforms which include four membrane bound (G1, G2, G3, and G4) and three soluble (G5, G6, G7) proteins; and restricted tissue distribution [29].

Under physiological circumstances, the expression of HLA-G molecules is mainly detected in placental trophoblast cells, but also in thymus, cornea, nail matrix, pancreas and erythroid and endothelial precursors [30]. HLA-G expression can be upregulated in various tissues 
under "pathological" conditions, such as in tumors, as well as after solid organ transplantation [31].

The immunosuppressive properties of both membranebound and soluble HLA-G proteins are probably due to their ability in:

(1) Inhibiting the activity and mediating apoptosis of cytotoxic CD8+ T cells and NK cells;

(2) Inhibiting proliferation of CD4+ T cells and driving them into an immunosuppressive profile;

(3) Inhibiting antigen presenting cells and B cells differentiation;

(4) Promoting a shift of Th1/Th2 balance toward Th2 polarization;

(5) Inducing regulatory T cells [29].

Secreted HLA-G5 contributes to the immunosuppressive functions of MSCs. As described previously in maternalfetal tolerance and tumor escape, HLA-G5 constitutes a potent molecule allowing MSCs to induce immunosuppression. Selmani et al. 2009, [31] demonstrated the expression of HLA-G5 proteins within the cytoplasm of MSCs, as well as in the supernatants from MSCs culture media. Other works reported that HLA-G expression is enhanced in IL10-treated monocytes [32]. In turn, HLA-G5 has been described previously as inducing a Th2 profile consisting of IL-10 secretion. HLA-G5 secreted by MSCs is also required for the ability of MSCs to expand functional CD4+ CD25 highFOXP3+ regulatory T cells [22].

\section{Transforming Growth Factor Beta (TGF- $\beta$ )}

One of the most important immunomodulatory cytokines produced and constitutively secreted by MSCs is transforming growth factor beta (TGF- $\beta$ ). Three TGF- $\beta$ isoforms have been described in mammalian species, including TGF- $\beta 1$, TGF- $\beta 2$, and TGF- $\beta 3$. TGF $\beta$ regulates multiple fundamental cellular functions, including proliferation, differentiation, migration, adhesion and apoptosis, that affect numerous biological processes such as development, wound healing, carcinogenesis, angiogenesis and immune responses [33]. TGF- $\beta 1$ is the prototype of TGF- $\beta$ s and is the predominant isoform expressed by immune system cells. Isoforms have different expression patterns and functional variations in vivo, but have relatively similar functions in experimental assays.

TGF- $\beta 1$ can suppress immune response either directly by inhibiting the function of immune cells, such as T cells, B cells, NK cells, and macrophages, or indirectly by inducing regulatory $T$ cells. One pathway by which TGF $\beta$ exerts its anti-proliferative effect on $\mathrm{T}$ lymphocytes is through blockade of the production of the T-cell mitogenic cytokine IL-2 [34]. TGF $\beta$ also inhibits cell proliferation through controlling the expression of cell cycle regulators, including up-regulation of cyclin-dependent kinase inhibitors (CKIs) p15, p21 and p27 and down-regulation of cell cycle-promoting factors, such as c-myc, cyclin D2 and cyclin E [35].

\section{Tool like Receptors (TLR)}

Toll-like receptors (TLR) are a class of molecules first discovered to play a role in body development and maintenance. TLR family has been shown to be of importance in the innate immune system for the recognition of pathogen associated molecular patterns (PAMPs) by immune cells, initiating a primary response toward invading pathogens and recruitment of the adaptive immune response [36]. Toll-like receptor activation triggers intracellular signaling pathways that lead to the induction of inflammatory cytokines, type I IFNs, and upregulation of co-stimulatory molecules leading to the activation of the adaptive immune response.

Functional TLR are expressed in adult MSCs and their activation by specific ligands regulates MSCs functions. Human MSCs have been reported to express TLR1, 2, 3, 4, 5, and 6 mRNA and TLR2, 3, 4, 7, and 9 proteins. The effect of TLR3 and TLR4 activation on MSC functions, including migration, differentiation, and immunomodulation, has been the particular focus of many recent studies, sometimes describing contradictory results [36].

Opitz et al. 2009, [37] reported that TLR3 and TLR4 engagement enhances the immunosuppressive properties of human BM-MSCs through the indirect induction of IDO1. While TLR3 priming results in production of antiinflammatory molecules such as IL4, IDO, or PGE2. TLR3- activated MSCs maintained the capacity to inhibit lymphocyte proliferation in vitro, while TLR4-primed MSCs activated T lymphocytes [38].

\section{Nitric Oxide (NO)}

Nitric oxide (NO) is an important signaling molecule and is involved in tissue homeostasis and immunoregulatory functions. iNOS is expressed by MSCs as a result of stimulation with IFN- $\gamma$ combined with TNF- $\alpha$, IL-1 $\alpha$, or IL-1 $\beta$. Immunosuppression is achieved when high levels of NO are released [39]. The mechanism of MSC-mediated immunosuppression is different in mice and humans. While murine MSCs use predominantly iNOS to control immune responses, human MSCs mainly utilize indoleamine 2,3-dioxygenase (IDO) [40]. NO production by MSCs is tightly regulated by proinflammatory cytokines and iNOS is its sole source. Two other NOS isoforms were undetectable in MSCs, with or without proinflammatory cytokine stimulation [41].

Induction of inducible nitric-oxide synthase (iNOS) by murine MSCs and production of nitric oxide was suggested to play a major role in T-cell proliferation inhibition [42]. Nitric oxide is a gaseous bioactive compound affecting macrophage and T-cell functions. iNOS is induced in mouse MSCs after activation by IFN $\gamma$ and TNF $\alpha$, IL- $1 \alpha$ or IL-1 $\beta$, and MSCs from iNOS-/- mice had a reduced ability to suppress T-cell proliferation. The expression level of iNOS mRNA in human MSCs was minimal, however, and secretion of nitric oxide by human MSCs was undetectable. Indeed, different mechanisms of immunosuppression exist in different species since human MSCs employ IDO as a major effector molecule whereas nitric oxide plays a critical role in mouse MSCs [43].

\section{Galectins (Gal)}

The family of human galectins is comprised of 15 members, of which 11 are expressed in human tissues [44] 
All galectins share carbohydrate recognition domains (CRDs) and preferentially bind to $\beta$-galactosides. Within the immune system, galectins are key players in the regulation of cellular homeostasis in both the innate and the adaptive immune system [45].

Within the immune system, galectins are expressed by virtually all immune cells, either constitutively or in an inducible fashion [46]. Also, they can be expressed by a spectrum of normal and tumor cells. As found in this study, Gal-3 is constitutively expressed by MSCs and upregulated in response to TLR-2 ligation. Gal-9 can be induced by IFN- $\gamma$ and interleukin-beta (IL-1 $\beta$ ) in various cell types and thus is upregulated in inflamed tissues. Gal9 must be released by cells to exert its immunoregulatory properties [46].

Sioud et al. [47] showed that Gal-3 can modulate MSCs immunosuppressive activity on T-lymphocyte proliferation. High levels of Gal-3 protein are found in MSCs culture supernatants; thus, it may participate in extra cellular matrix (ECM)-cell interactions and modulation of surrounding immune cells.

\section{Heat Shock Proteins (HSP)}

Heat shock proteins form an ancient, primary system for "intracellular self-defense". Most of the HSP are molecular chaperones. Chaperones have been defined as proteins that bind to and stabilize an otherwise unstable conformer of another protein - and, by controlled binding and release, facilitate its correct fate in vivo: be it folding, oligomeric assembly, transport to a particular subcellular compartment, or disposal by degradation [48].

HSP exhibit immunomodulatory functions that further promote immune responses by acting as pro-inflammatory cytokines and by mediating regulatory immune responses. Understanding the mechanisms of HSP involvement in the balance of autoimmunity versus tolerance is essential for the establishment of HSP as potential therapeutic tools for the treatment of different autoimmune diseases [49]. Findings from in vitro studies have shown that HSP, including Hsp70, Hsp90, gp96 (ER Hsp90), are released from necrotic cells (although not by apoptotic cells) after trauma or severe stress, and these HSP act as danger signals, promoting DCs maturation and exerting immunostimulatory functions [50].

Recent works on cell exposure to biophysical stimuli provide potential alternatives for boosting MSCs paracrine actions. As the Hsp family of molecules has been implicated in cytoprotection and immunomodulation, laser preconditioning of stem cells could provide a means of increasing their paracrine actions [51].

\section{Conclusions}

MSCs have potential to differentiate into mesenchymal lineages, homing or migration capacity and immunoregulatory properties. These characteristics makes MSCs an effective tool in the treatment of several pathologies, among them, immune diseases. MSCs have immunomodulatory properties, which are directly mediated by cell-cell contact or through the secretion of vesicles called exosomes. These lipidic bilayer vesicles transport various substances, such as TGF-beta, PGE2, HLA-G, IDO, Gal, among others that have actions on the immune system.

However many questions remain to be addressed in order to provide better ways to control and optimize the immune response for the benefit of the patient. This implies a better understanding of the underlying mechanisms of immunoregulation.

\section{References}

[1] J. Reiser, XY. Zhang, VF. La Russa, "Potential of mesenchymal stem cells in gene therapy approaches for inherited and acquired diseases”, Expert Opin Biol Therapy, 2005.

[2] EM. Horwitz, K. Le Blanc, M. Dominici, I. Mueller, I. SlaperCortenbach, Marini FC, "Clarification of the nomenclature for MSC: The International Society for Cellular Therapy position statement”, Cytotherapy, 7, (5), 393-5, 2005.

[3] M. Di Nicola, C. Carlo-Stella, M. Magni. "Human bone marrow stromal cells suppress $\mathrm{T}$ lymphocyte proliferation induced by cellular or nonspecific mitogenic stimuli”, Blood, 99: 3838-3843, 2002.

[4] RE. Newman, D. Yoo, MA. LeRoux, A. Danilkovitch-Miagkova. "Treatment of Inflammatory Diseases with Mesenchymal Stem Cells”, Inflammation \& Allergy - Drug Targets, 8, 110-123, 2009.

[5] K. Le Blanc, L. Tammik, B. Sundberg, SE. Haynesworth, O. Ringden. "Mesenchymal stem cells inhibit and stimulate mixed lymphocyte cultures and mitogenic responses independently of the major histocompatibility complex”, Scand J Immunol, 57:11-20, 2003.

[6] WT. Tse, JD. Pendleton, WM. Beyer, MC. Egalka, EC. Guinan. "Suppression of allogeneic T-cell proliferation by human marrow stromal cells: implications in transplantation”, Transplantation, 75: 389-397, 2003.

[7] I. Rasmusson, O. Ringden, BL. Sundberg. K. Blanc. "Mesenchymal stem cells inhibit lymphocyte proliferation by mitogens and alloantigens by different mechanisms", Exp Cell Res, 305:33-41, 2005.

[8] C. Coquerelle, M. Moser, "DC subsets in positive and negative regulation of immunity”, Immunol. Rev. 234:317-334, 2010.

[9] Lei Chen, Wei Zhang, Han Yue, Qin Han, Bin Chen, Mingxia Shi, Jing Li, Binzong Li, Shengguo You, Yufang Shi, and Robert Chunhua Zhao. "Effects of Human Mesenchymal Stem Cells on the Differentiation of Dendritic Cells from CD34+ Cells”, Stem Cells and Development, 16(5): 719-732, 2007.

[10] L. Bai, DP. Lennon, V. Eaton, K. Maier, AI. Caplan, SD. Miller, RH. Miller. "Human bone marrow-derived mesenchymal stem cells induce Th2-polarized immune response and promote endogenous repair in animal models of multiple sclerosis”, Glia, 15; 57(11):1192-203, 2009.

[11] TL. Bonfield, MF. Koloze, DP. Lennon, B. Zuchowski, SE. Yang, AL. Caplan. "Human Mesenchymal Stem Cells Suppress Chronic Airway Inflammation in the Murine Ovalbumin Asthma Model”, Am J Physiol Lung Cell Mol Physiol, 2010.

[12] DL. Roelen, BJ. van der Mast, PS. in't Anker, C. Kleijburg, M. Eikmans, E. van Beelen, GM. de Groot-Swings, WE. Fibbe, HH. Kanhai, SA. Scherjon, FH. Claas, "Differential immunomodulatory effects of fetal versus maternal multipotent stromal cells”, Human Immunology, 70(1):16-23, 2009.

[13] T. Yuan, L. Zhang, L. Feng, H. Fan, X. Zhang. “Chondrogenic differentiation and immunological properties of mesenchymal stem cells in collagen type I hydrogel”, Biotechnol Prog, 2010.

[14] GM. Spaggiari, H. Abdelrazik, F. Becchetti, L. Moretta. "MSCs inhibit monocyte-derived DC maturation and function by selectively interfering with the generation of immature DCs: central role of MSC-derived prostaglandin E2”, Blood, 113:65766583, 2009.

[15] R. Blazquez, F. Sanchez-Margallo, O. de la Rosa, W. Dalemans, V. Álvarez, R. Tarazona, JG. Casado. "Immunomodulatory Potential of Human Adipose Mesenchymal Stem Cells Derived Exosomes on in vitro Stimulated T Cells”, Front Immunol.; 5:556, 2014.

[16] B. Yu, X. Zhang, X. Li. "Exosomes derived from mesenchymal stem cells”, Int J Mol Sci, 7; 15(3):4142-57, 2014. 
[17] F. Morandi, L. Raffaghello, G. Bianchi, F. Meloni, A. Salis, E. Millo, S. Ferrone, V. Barnaba, V. Pistoia. "Immunogenicity of human mesenchymal stem cells in HLA-class I-restricted T-cell responses against viral or tumor-associated antigens”, Stem Cells, 26(5):1275-87, 2008.

[18] RP. Phipps, SH. Stein, RL. Roper. "A new view of prostaglandin E regulation of the immune response”, Immunol. Today 12: 349352, 1991.

[19] L. Gao, WE. Zackert, JJ. Hasford, ME. Danekis, GL. Milne, C. Remmert, J. Reese, H. Yin, HH. Tai, SK. Dey, et al., "Formation of prostaglandins E2 and D2 via the isoprostane pathway: a mechanism for the generation of bioactive prostaglandins independent of cyclooxygenase”, J. Biol. Chem. 278: 2847928489, 2003.

[20] LJ. Crofford, "COX-1 and COX-2 tissue expression: Implications and predictions”, J Rheumatol Suppl, 49:15-19, 1997.

[21] R. Meisel, A. Zibert, M. Laryea, "Human bone marrow stromal cells inhibit allogeneic T-cell responses by indoleamine 23dioxygenase mediated tryptophan degradation”, Blood. 103:46194621, 2004.

[22] S. Aggarwal, MF. Pittenger, "Human mesenchymal stem cells modulate allogeneic immune cell responses”, Blood, 105:18151822, 2005.

[23] P. Kalinski, "Regulation of immune responses by prostaglandin E2”, J Immunol, Jan 1; 188 (1):21-8, 2012.

[24] GM. Spaggiari, A. Capobianco, S. Becchetti, MC. Mingari, L. Moretta. "Mesenchymal stem cell natural killer cell interactions: evidence that activated NK cells are capable of killing MSCs whereas MSCs can inhibit IL-2-induced NK-cell proliferation”, Blood. 107:1484-1490, 2006.

[25] H. Soliman, B. Rawal, J. Fulp, JH. Lee, A. Lopez, MM. Bui, F. Khalil, S. Antonia, HG. Yfantis, DH. Lee, TH. Dorsey, S. Ambs. "Analysis of indoleamine 2-3 dioxygenase (IDO1) expression in breast cancer tissue by immunohistochemistry”, Cancer Immunol Immunother, 62(5):829-37, 2013.

[26] C. Liang, SL. Chen, M. Wang, WJ. Zhai, Z. Zhou, AM. Pang, SZ. Feng, MZ. Han. "Synergistic immunomodulatory effects of interferon-gamma and bone marrow mesenchymal stem cells", Zhonghua Xue Ye Xue Za Zhi, 34:213-216, 2013.

[27] WT. Hsu, CH. Lin, BL. Chiang, HY. Jui, KK. Wu, CM. Lee. "Prostaglandin E2 potentiates mesenchymal stem cell-induced IL$10+$ IFN $-\gamma+\mathrm{CD} 4+$ regulatory $\mathrm{T}$ cells to control transplant arteriosclerosis”, J Immunol, 190:2372-2380, 2013.

[28] AL. Mellor, DH. Munn. "IDO expression by dendritic cells: tolerance and tryptophan catabolism”, Nat Rev Immunol, 4 (10):762-74, 2004.

[29] E. Fainardi, M. Castellazzi, M. Stignani, F. Morandi, G. Sana, R. Gonzalez, V. Pistoia, OR. Baricordi, E. Sokal, J. Peña. "Emerging topics and new perspectives on HLA-G”, Cell Mol Life Sci. 2010.

[30] C. Menier, N. Rouas-Freiss, B. Favier, J. LeMaoult, P. Moreau, ED. Carosella, "Recent advances on the non-classical major histocompatibility complex class I HLA-G molecule”, Tissue Antigens, 75: 201-206, 2010.

[31] Z. Selmani, A. Naji, I. Zidi, B. Favier, E. Gaiffe, L. Obert, C. Borg, P. Saas, P. Tiberghien, N. Rouas-Freiss, ED. Carosella, F. Deschaseaux, "Human Leukocyte Antigen-G5 Secretion by Human Mesenchymal Stem Cells Is Required to Suppress T Lymphocyte and Natural Killer Function and to Induce CD4+CD25highFOXP3+ Regulatory T Cells”, Stem Cells, 26: 212-222, 2008.

[32] P. Moreau, F. Adrian-Cabestre, C. Menier et al. "IL-10 selectively induces HLA-G expression in human trophoblasts and monocytes", Int Immunol; 11: 803-811, 1999.

[33] MO. Li, YY. Wan, S. Sanjabi, AK. Robertson, RA. Flavell. "Transforming growth factor-beta regulation of immune responses”, Annu Rev Immunol, 24:99-146, 2006.

[34] T. Brabletz, I. Pfeuffer, E. Schorr, F. Siebelt, T. Wirth, E. Serfling. "Transforming growth factor beta and cyclosporin A inhibit the inducible activity of the interleukin-2 gene in T cells through a noncanonical octamer-binding site”, Mol Cell Biol, 13 (2):115562, 1993.

[35] A. Nasef, A. Chapel, C. Mazurier, S. Bouchet, M. Lopez, N. Mathieu, et al. "Identification of IL-10 and TGF-beta transcripts involved in the inhibition of T-lymphocyte proliferation during cell contact with human mesenchymal stem cells", Gene Expr, 13(4-5):217-26, 2007.

[36] M. Fischer, M. Ehlers, Toll-like Receptors in Autoimmunity. Annals of the New York Academy of Sciences, 1143: 21-34, 2008

[37] CA. Opitz, U. M Litzenburger, C. Lutz, TV. Lanz, I. Tritschler, J. Köppel, WK. Aicher, M. Weller, W. Wick, M. Platten, “Toll-like receptor engagement enhances the immunosuppressive properties of human bone marrow-derived mesenchymal stem cells by inducing indoleamine-2,3-dioxygenase-1via interferon-beta and proteinkinase”, Stem Cells 27, 909-919, 2009.

[38] O. DelaRosa, W. Dalemans, E. Lombardo. "Toll-Like Receptors as Modulators of Mesenchymal Stem Cells”, Front Immunol, 3: 182, 2012.

[39] G. Ren, L. Zhang, X. Zhao, G. Xu, Y. Zhang, AI. Roberts, RC. Zhao, Y. Shi. "Mesenchymal stem cell-mediated immunosuppression occurs via concerted action of chemokines and nitric oxide”, Cell Stem Cell, 7; 2(2):141-50, 2008.

[40] I. Molendijk, M. Duijvestein, AE. van der Meulen-de Jong, WK. van Deen, M. Swets, DW. Hommes, HW. Verspaget. "Immunomodulatory Effects of Mesenchymal Stromal Cells in Crohn's Disease”. Journal of Allergy; Volume, 2012.

[41] W. Li, G. Ren, Y. Huang, J. Su, Y. Han, J. Li, X. Chen, K. Cao, Q. Chen, P. Shou, L. Zhang, Z-R. Yuan, AI. Roberts, S. Shi, AD. Le Y. Shi. "Mesenchymal stem cells: a double-edged sword in regulating immune responses”, Cell Death and Differentiation, 19, 1505-1513, 2012.

[42] S. Ghannam, C. Bouffi, F. Djouad, C. Jorgensen D. Noël. "Immunosuppression by mesenchymal stem cells: mechanisms and clinical applications”, Stem Cell Research \& Therapy, 1:2, 2010.

[43] C. Bouffi, C. Bony, G. Courties, Jorgensen C, Noel D, "IL-6dependent PGE2 secretion by mesenchymal stem cells inhibits local inflammation in experimental arthritis”, PLoS One, 7; 5(12), 2010.

[44] Thijssen, V. L., Poirier, F., LG. Baum, AW. Griffioen, “Galectins in the tumor endothelium: opportunities for combined cancer therapy”, Blood. 110: 2819-2827, 2007.

[45] RY. Yang, GA. Rabinovich, FT. Liu, "Galectins: structure, function and therapeutic potential”, Expert Rev. Mol. Med. 10: e17, 2008.

[46] F. Gieseke, A. Kruchen, N. Tzaribachev, F. Bentzien, M. Dominici, I. Muller. Proinflammatory stimuli induce galectin-9 in human mesenchymal stromal cells to suppress T-cell proliferation. Eur. J. Immunol. 43: 2741-2749, 2013.

[47] M. Sioud, A. Mobergslien, A. Boudabous, Y. Fløisand, "Evidence for the Involvement of Galectin-3 in Mesenchymal Stem Cell Suppression of Allogeneic T-Cell Proliferation”, Scandinavian Journal of Immunology, 71: 267-274, 2010.

[48] FU. Hartl, "Molecular chaperones in cellular protein folding", Nature, 381, 571-580, 1996.

[49] MJ. Mansilla, X. Montalban, C. Espejo. "Heat shock protein 70: roles in multiple sclerosis”, Mol Med, 7; 18: 1018-28, 2012.

[50] S. Basu, RJ. Binder, R. Suto, KM. Anderson, PK. Srivastava. "Necrotic but not apoptotic cell death releases heat shock proteins, which deliver a partial maturation signal to dendritic cells and activate the NF-kappa B pathway”, Int Immunol 12: 1539-46, 2000.

[51] D. Cizkova, J. Rosocha, I. Vanicky, J. Radonak, J. Galik, M. Cizek. "Induction of mesenchymal stem cells leads to HSP72 synthesis and higher resistance to oxidative stress", Neurochem. Res, 31(8):1011-1020, 2006. 